\title{
Efficacy of Mycobacterium Phlei Cell Wall-Nucleic Acid Complex (MCNA) in BCG-Unresponsive Patients
}

\author{
Roger $\mathrm{Li}^{\mathrm{a}}$, John Amrhein ${ }^{\mathrm{b}}$, Zvi Cohen ${ }^{\mathrm{c}}$, Monique Champagne ${ }^{\mathrm{c}}$ and Ashish M. Kamat ${ }^{\mathrm{a}, *}$ \\ ${ }^{a}$ Department of Urology, The University of Texas MD Anderson Cancer Center, Houston, TX, USA \\ ${ }^{\mathrm{b}}$ McDougall Scientific, Toronto, ON, Canada \\ ${ }^{\mathrm{c}}$ Telesta Therapeutics Inc., Saint-Laurent, QC, Canada
}

\begin{abstract}
.
Background: We have previously reported the results of a prospective multi-institutional study on the efficacy of MCNA in patients who recurred after intravesical BCG treatment [1]. Since that publication, a new standardized definition for BCG-unresponsiveness has been established [2].

Objective: We re-analyzed the oncologic outcomes following intravesical MCNA in patients classified as BCG-unresponsive according to the new definition.

Methods: For this analysis, we focused on the enrolled patients who satisfied the requirements for BCG Unresponsiveness: i.e. adequate BCG treatment (at least 5/6 induction and 2/3 maintenance instillations) and high grade tumor within 6 months of prior BCG. Treatment course included 6 weekly intravesical instillations of $8 \mathrm{mg}$ MCNA followed by 3 weekly instillations at months 3, 6, 12, 18, and 24. Followup assessments included cystoscopy, urine cytology and biopsy. Patients absent of high grade disease confirmed by central review of biopsy were deemed disease-free.

Results: Of the 129 patients enrolled, 94 (68 CIS with/without papillary tumors, 26 papillary only tumors) fit the criteria for the new BCG Unresponsive definition. Overall, disease free survival (DFS) for all BCG unresponsive patients was $48.9 \%$ (95\% CI 38.0-59.0\%) at 6 months, $34.8 \%$ (95\% CI 24.7-45\%) at 1 year and $28.3 \%(15.7-34.3 \%)$ at 2 years post induction. In the group with papillary tumors, DFS measured at months 6,12 , and 24 were: $61.2 \%(38.2-77.8 \%), 61.2(38.2-77.8 \%)$, and $50.1 \%(27.5-69 \%)$. In the CIS-containing group, the corresponding DFS were: $44.8 \%(32.3-56.4 \%), 26.5 \%(16.3-37.9 \%)$, and $16.6 \%(8.6-26.9 \%)$, respectively.

Conclusions: For patients who are BCG Unresponsive, MCNA has the potential to render $26.5 \%$ of patients with CIS and $61.2 \%$ of patients with papillary tumors disease-free for at least 1 year with an intact bladder. The higher efficacy noted in the true BCG-unresponsive cohort than was previously reported with all-comers emphasizes the importance of having clearly defined criteria for clinical trials investigating new intravesical therapies after BCG failure.
\end{abstract}

Keywords: Bladder cancer, MCNA, BCG, BCG-unresponsive

\footnotetext{
${ }^{*}$ Correspondence to: Ashish M. Kamat, Department of Urology, The University of Texas MD Anderson Cancer Center, 1515 Holcombe Blvd, Unit 1373, Houston, TX 77030, USA. Tel.: +1 713 792 3250; Fax: +1 713794 4824; E-mail: akamat@mdanderson. org.
} 
MCWE Mycobacterium phlei cell wall extract IBCG International Bladder Cancer Group

MIBC muscle invasive bladder cancer

\section{INTRODUCTION}

Since its inception in 1976 [3], intravesical Bacillus Calmette-Guerin (BCG) has been the most effective immunotherapy for intermediate and high risk non-muscle invasive bladder cancer (NMIBC). However, not all patients respond to therapy and inoculation with live (albeit attenuated) bacteria in BCG can sometimes cause serious adverse events. As such, investigations have been carried out to assess the antineoplastic ability of nonpathogenic BCG cell wall components [4]. Mycobacterium phlei cell wallnucleic acid complex (MCNA) is one such agent with both immunomodulatory and antineoplastic activities [5]. Its efficacy and safety in treating high risk NMIBC had been well documented in two international, multi-centered trials $[1,6]$.

The results from a prospective multi-institutional study on the efficacy of MCNA in patients who recurred after intravesical BCG treatment were reported in 2015. In that study, DFS at 1 and 2 years were $25 \%$ and $19 \%$, respectively [1]. Unfortunately, as the definition of BCG failure was not standardized, the heterogeneous study population made interpretation of the results difficult and the agent was deemed as not having met its primary endpoint [7]. This highlights the need for a well-accepted definition of BCG unresponsiveness and clear study endpoints.

To resolve this issue, a group of U.S. experts convened at Genitourinary Cancers Symposium in 2015 to clarify disease states following intravesical BCG treatment [8]. This was further consolidated by the International Bladder Cancer Group (IBCG) in their white paper on 'Definitions, End Points, and Clinical Trial Designs for Non-Muscle-Invasive Bladder Cancer'[2]. This definition has been adopted by single-armed trials developed and being developed in the BCG failure setting. Thus, to be considered BCG Unresponsive, the patient should have high grade tumor recurrence within 6-9 months from the last exposure to BCG despite adequate BCG treatment (at least five of six induction instillations and two of three maintenance doses of BCG) [2]. With this new definition in mind, we re-analyzed data from clinical trial NCT00406068 [1], focusing on the outcomes in the BCG Unresponsive patients. By restricting the analysis to this standardized population, we aim to re-interpret the efficacy of MCNA and provide a benchmark for meaningful comparisons.

\section{MATERIALS AND METHODS}

As previously reported, 129 patients with high grade NMIBC recurring after BCG treatment were recruited from 25 sites in North America [1]. For the purposes of the current study, only clinical outcomes from the patients meeting the criteria for BCG Unresponsive were included in analysis. MCNA administration comprised of 3 phases, including 1) a 6 -week induction phase ( $8 \mathrm{mg}$ in $50 \mathrm{~mL}), 2)$ a maintenance phase in which patients were to receive 3 or 6 (re-induction) weekly MCNA instillations at month 3 followed by 3 weekly instillations each at months $6,12,18$ and 24,3 ) a followup phase with evaluations every 6 months (Fig. 1). Endoscopic evaluation and urine cytology were performed at each followup time point, along with transurethral resection and/or biopsy for all apparent or suspicious areas of malignancy. All patients underwent mandatory biopsy at 6-month evaluation.

Recurrence was defined as pathologically confirmed diagnosis of high grade urothelial carcinoma, while progression was defined as pathologically confirmed diagnosis of MIBC, metastatic bladder cancer or bladder cancer death. All patients with recurrence and/or progression were withdrawn from the study, while disease-free patients continued on maintenance treatment. Patients found to have low grade recurrences during surveillance were not deemed treatment failures per the new IBCG definition.

Statistical analysis was carried out using $\operatorname{SAS}^{\circledR}$, Version 9.2 for Windows. Continuous variables were reported using mean values and were analyzed using the Student's $t$-test. Categorical variables were reported using frequency values and analyzed using the Chi-squared test. Kaplan-Meier analysis was used to generate disease free survival curves and the difference among survival curves was examined with $\log$-rank test. $P$ value of $<0.05$ was deemed statistically significant.

\section{RESULTS}

A total of 94 out of the 129 patients met the BCG unresponsive definition. Baseline characteristics of the BCG unresponsive group were similar to those of the rest of the study subjects (Table 1). In the BCG unresponsive group, 68 (72.3\%) had CIS with 


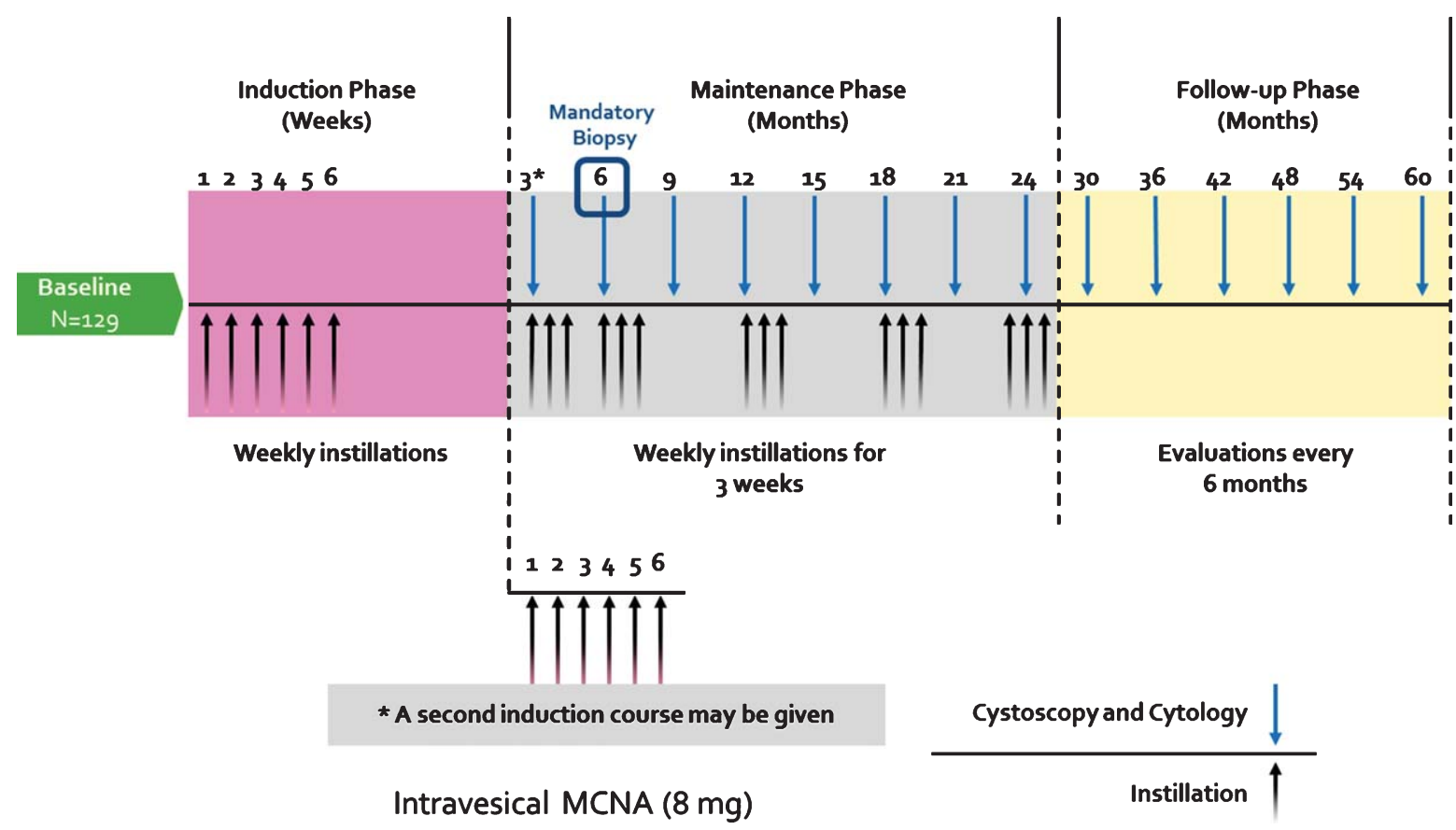

Fig. 1. MCNA instillation and followup protocol. A 6-weekly induction phase $(8 \mathrm{mg}$ in $50 \mathrm{~mL})$ was followed by a maintenance phase in which patients received 3 or 6 (re-induction) weekly MCNA instillations at month 3 followed by 3 weekly instillations each at months 6 , 12, 18 and 24, and 36. During the follow-up phase, cystoscopic evaluations and urine cytologies were conducted every 6 months. (Figure adopted from Morales et al. [1]).

$(24.4 \%)$ or without $(47.8 \%)$ concomitant papillary disease. On average, the BCG unresponsive patients received 16.1 doses of $\mathrm{BCG}$ prior to study enrollment compared to 6.1 received by the rest of the study subjects $(p<0.0001)$.

The DFS for BCG unresponsive patients was $48.9 \%(95 \%$ CI $38.0-59.0 \%)$ at 6 months, $34.8 \%$ $(95 \%$ CI $24.7-45 \%)$ at 1 year and $28.3 \%$ $(15.7-34.3 \%)$ at 2 years post induction, compared to $38.4 \%$ (95\% CI $29.8-46.9 \%$ ), $25.0 \%$ (17.7-30\%) and $19 \%(12.6-26.5 \%)$ reported in the entire study cohort [1]. Patients with papillary only disease at baseline had DFS of $61.2 \%$ (95\% CI 38.2-77.8\%) at 6 months, $61.2 \%$ (95\% CI 38.2-77.8\%) at 1 year, and $50.1 \%$ (95\% CI 27.5-69.0\%) at 2 years. Patients with CIS with/without concurrent papillary disease at baseline had DFS of $44.8 \%$ (95\% CI 32.3-56.4\%) at 6 months, $26.5 \%(95 \%$ CI $16.3-37.9 \%)$ at 1 year, and $16.6 \%$ (95\% CI 8.6-26.9\%) at 2 years (Table 2).

The median DFS for BCG unresponsive patients increased from 175 to 182 days, compared to 167 days for the rest of the study subjects. In patients who were disease free at 1 year, the durable DFS stretched to 34.0 months. Compared to the rest of the subjects in the study [1], BCG unresponsive patients had a non-statistically significant improvement in DFS to intravesical MCNA ( $p=0.273$ ) (Fig. 2a). The improvement was seen in both subgroups with papillary only disease and CIS containing tumors at baseline (Fig. 2b, c).

Overall, 42/94 patients (47.9\%) underwent radical cystectomy, 15 patients (16.0\%) progressed to MIBC, 11 patients $(11.7 \%)$ developed metastasis, and $18(19.1 \%)$ succumbed to their disease. A total of $27(28.7 \%)$ patients were unresponsive to MCNA treatment, with recurrence found at the 6 month followup. Of these patients, $70.4 \%$ underwent cystectomy, $25.9 \%$ progressed to MIBC, $14.8 \%$ developed metastasis, and $25.9 \%$ succumbed to their disease. Median time to cystectomy was 10 months from the first dose of MCNA administration.

\section{DISCUSSION}

Our results show that MCNA has measurable activity in patients who are deemed to be BCG Unresponsive based on criteria established in 2016 for this disease state, which is currently being used in prospective single-armed studies. 


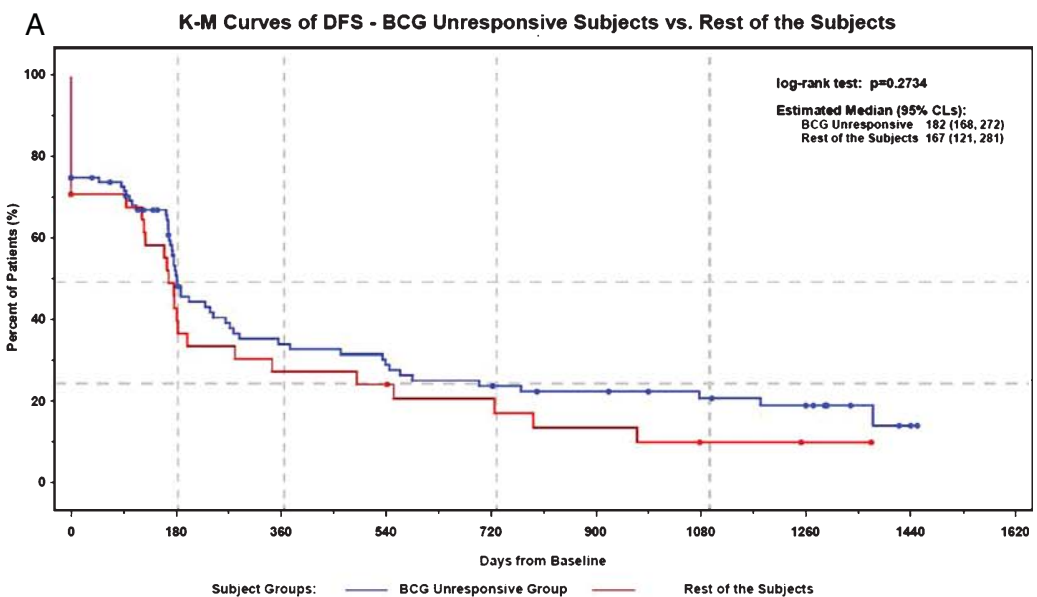

Censored observations are in dicated by dots

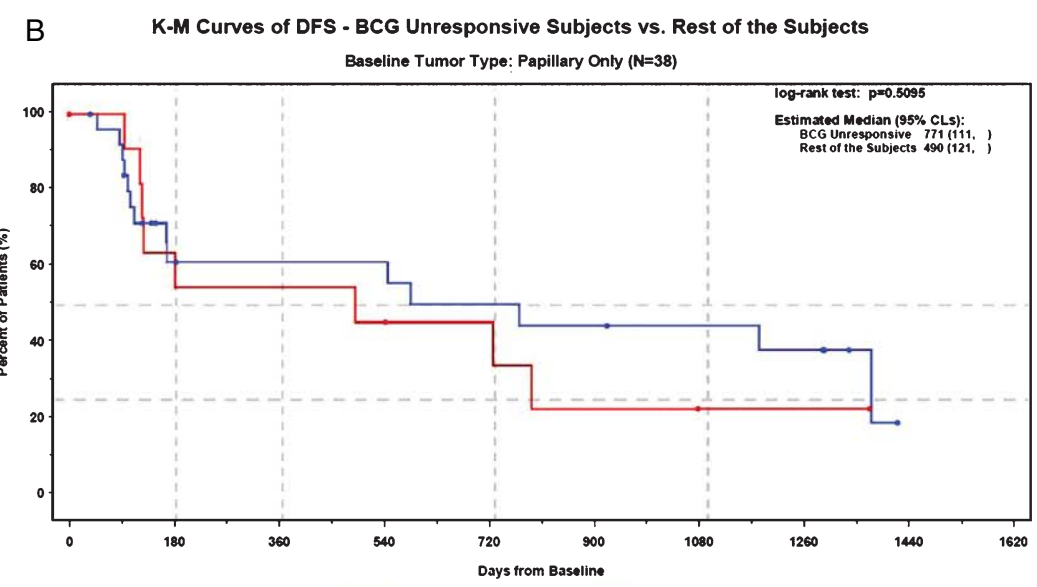

Subject Groups: $\quad$ BCG Unresponsive Group $\quad$ Rest of the Subjects

Censored observations are indicated by dots

C K-M Curves of DFS - BCG Unresponsive Subjects vs. Rest of the Subjects

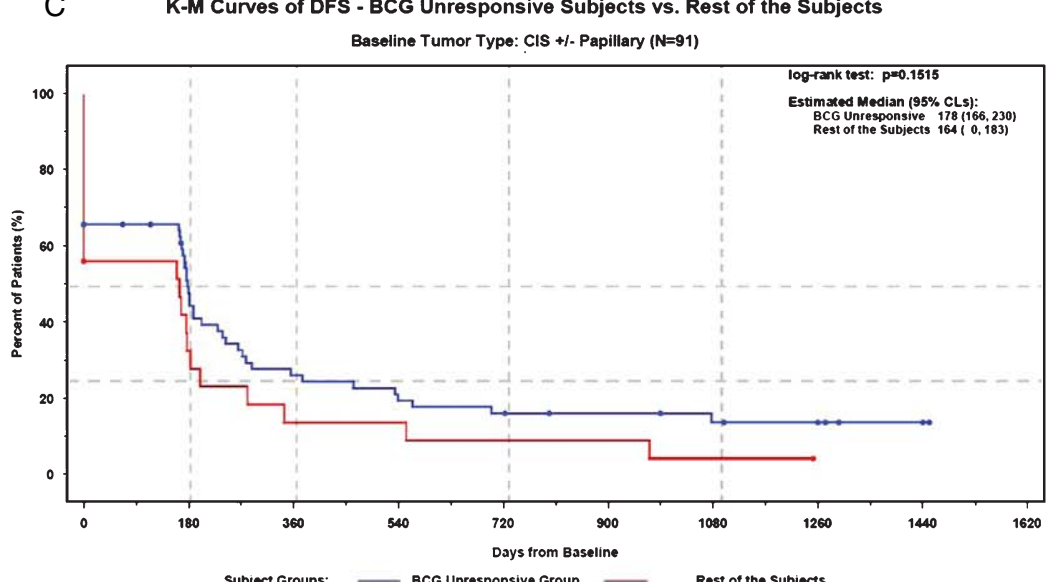

Censored observations are indicated by dots

Fig. 2. Kaplan-Meier curves showing non-statistically significant improvement in DFS after intravesical MCNA treatment in the BCG Unresponsive patients. This trend was seen in all patients (a), those with papillary only disease at baseline (b), and those with CIS with/without concurrent papillary disease at baseline (c). 
Table 1

Baseline characteristics

\begin{tabular}{lcccc}
\hline & BCG Unresponsive $n=94$ & Rest of subjects $n=35$ & Total $n=129$ & $p$ value \\
\hline Mean age (SD) & $67.9(11.0)$ & $70.1(11.8)$ & $68.5(11.2)$ & 0.706 \\
Gender (\%) & $65(69.1)$ & $30(85.7)$ & $95(73.6)$ & 0.058 \\
$\quad$ Male & $29(30.9)$ & $5(14.3)$ & $34(26.4)$ & 0.690 \\
Female & & & & \\
Baseline tumor type (\%) & $45(47.9)$ & $14(40)$ & $59(45.7)$ & \\
CIS only & $26(27.7)$ & $12(34.3)$ & $38(29.5)$ & \\
Papillary only & $23(24.5)$ & $9(25.7)$ & $32(24.8)$ & \\
Papillary and CIS & & & & \\
Papillary tumor stage (\%) & $42(85.7)$ & $14(66.7)$ & $56(80.0)$ & \\
Ta & $4(8.2)$ & $5(23.8)$ & $9(12.9)$ & \\
T1 & $3(6.1)$ & $2(9.5)$ & $5(7.1)$ & \\
T2-4 & $17(34.7)$ & $2(9.5)$ & $19(27.1)$ & \\
Baseline Grade (\%) & $30(61.2)$ & $17(80.9)$ & $47(67.1)$ & \\
Low & $2(8.2)$ & $2(9.5)$ & $4(5.7)$ & \\
High & $16.1(8-33)$ & $6.1(5-7)$ & $13.4(5-33)$ & $<\mathbf{0 . 0 0 0 1}$ \\
Missing & & & & \\
Mean \# BCG (Range) & & & & \\
\hline
\end{tabular}

Table 2

Kaplan-meier estimates of disease free survival

\begin{tabular}{lcccccr}
\hline & $n$ & \multicolumn{5}{c}{ Disease Free Survival Rate Estimate (\%) (95\% CI's) } \\
\cline { 3 - 7 } & & 3 Months & 6 Months & 1 Year & 18 Months & 2 Years \\
\hline CIS & 68 & $66.2(53.6,76.1)$ & $44.8(32.3,56.4)$ & $26.5(16.3,37.9)$ & $19.9(11.0,30.6)$ & $16.6(8.6,26.9)$ \\
CIS Only & 45 & $64.4(48.7,76.5)$ & $49.6(34.0,63.4)$ & $29.7(16.8,43.9)$ & $22.3(11.1,35.9)$ & $\mathrm{n} / \mathrm{a}$ \\
CIS + Papillary & 23 & $69.6(46.6,84.2)$ & $34.8(15.8,54.6)$ & $19.9(6.3,39)$ & $14.9(3.7,33.2)$ & $14.9(3.7,33.2)$ \\
Papillary Only & 26 & $92.0(71.6,97.9)$ & $61.2(38.2,77.8)$ & $61.2(38.2,77.8)$ & $55.6(32.6,73.6)$ & $50.1(27.5,69.0)$ \\
Overall & 94 & $73.3(63.1,81.1)$ & $48.9(38.0,59.0)$ & $34.8(24.7,45.0)$ & $28.3(19.0,38.4)$ & $24.5(15.7,34.3)$ \\
\hline
\end{tabular}

While many patients respond to intravesical immunotherapy with BCG, BCG failure is unfortunately an often encountered scenario in urologic practice. Although radical cystectomy remains the gold standard in patients fit for surgery, its potential risks, morbidity and negative impact on quality of life need to be considered $[9,10]$. Many agents have been proposed and studied as alternatives to cystectomy in this setting. However, their efficacy cannot be reliably compared due to the heterogeneous population of BCG failure patients and various definitions used. To guide uniform clinical trial design, the term BCG Unresponsive has recently been proposed and adopted. This term defined a cohort at highest risk for cancer relapse and progression, for whom additional BCG therapy is futile and experimental single-armed trials can be conducted. For such trials, initial complete response rates (for CIS) or recurrence-free rates (for papillary tumors) of 50\% at 6 months, $30 \%$ at 1 year and $25 \%$ at 18 months can be considered clinically meaningful $[2,11]$.

The results of the current analysis of MCNA response in the BCG Unresponsive cohort (48.9\% at 6 months, $34.8 \%$ at 1 year and $28.3 \%$ at 2 years) falls within the accepted threshold. Furthermore, it compares favorably with Valrubicin (the only FDA approved agent for CIS patients after BCG failures), which resulted in $21 \%$ complete response rate at 6 months and $17 \%$ at 1 year [12]. Treatment with gemcitabine has been reported as an alternative. In one study of 47 patients with high grade disease recurrence after BCG, DFS at 1 year and 2 year were $28 \%$ and $21 \%$, respectively [13]. A phase II trial using nab(nanoparticle albumin bound)-paclitaxel in 28 patients with a minimum of 1 course of BCG previously demonstrated 1 year and 21 month DFS of $35.7 \%$ and $25 \%$, respectively [14]. Other studies using INF- $\alpha /$ BCG [15] and thermochemotherapy [16] reported much higher 2-year response rates than those observed in the current study. However, many of the patients included in these studies would not qualify as BCG-unresponsive either due to insufficient BCG treatment prior to relapse or because of their non-high grade recurrence. This discrepancy in patient characteristics across studies further highlights the importance of adhering to the IBCG consensus definition for clinical trial design and analysis. For example, trials which may include patients who are not cystectomy candidates (i.e. low grade recurrence, inadequate BCG treatment) would 
artificially inflate response rate but not contribute meaningfully to the development of treatments for BCG failure.

Along the same lines, exclusion of low grade recurrence as treatment failure is an important consideration for such trials since low grade recurrences rarely causes metastasis [9]. In a large institutional retrospective study, Mmeje et al. showed a lack of cancer progression associated with low grade recurrence found at the 3-month cystoscopy [17]. As such, patients with low grade tumors after intravesical therapy can be safely managed conservatively and are not regarded as treatment failure by either the latest EAU or AUA NMIBC guidelines [9, 10]. The characterization of these patients as treatment failures previously may have underestimated the efficacy of other intravesical therapeutic agents.

One caveat when evaluating any therapy for BCG Unresponsive patients remains the high risk of understaging patients with bladder cancer and the inadequacies of current imaging and staging modalities. In a lower risk, BCG naive population, Oddens et al. reported progression and metastasis rates of $8.0 \%$ and $4.9 \%$, respectively with treatment of induction and maintenance BCG [18]. In a higher risk group having failed a minimum of 2 prior courses of BCG, Skinner et al. reported progression and metastasis rates of $4.3 \%$ and $0 \%$, respectively, during the course of salvage intravesical gemcitabine [13]. However, the same authors reported a high cystectomy rate of $36 \%$. In the MCNA study, 13 patients opted for further intravesical therapy even after MCNA treatment failure, which may have contributed to the $16 \%$ progression and $11.7 \%$ metastatic rates observed. As with using other conservative strategies in the BCG failure setting, high vigilance for progression and metastasis needs to be maintained throughout the treatment course, with liberal use of salvage extirpative surgery as necessary. Unfortunately, the physical frailty and unwillingness of the patients often play a major role in directing the treatment course.

Interestingly, in this homogeneous group with high grade bladder cancer recurrence after having undergone adequate BCG treatment, higher responsive rates to MCNA were observed than in the original report. This is consistent with the previous finding that patients who received maintenance or second induction BCG had higher DFS after MCNA than those only having undergone one course of induction [1]. This finding is unique among salvage therapies for BCG failure. Persistent failure after multiple doses of intravesical BCG had been shown to adversely affect outcomes following other salvage intravesical treatment [15]. Although speculative, one hypothesis is that the patients having received higher doses of intravesical BCG prior to study enrolment were better immunologically primed, leading to enhanced tumoricidal activities brought on by a more robust immune response [5].

The current study demonstrates the importance of implementing a uniform definition and clearly defined inclusion criteria for patients in trials designed to evaluate efficacy of agents after BCG therapy for NMIBC. This is especially relevant since such studies are single-armed studies, due to the fact that there is no true effective therapy to serve as a control arm other than radical surgery.

\section{CONCLUSION}

For patients who are BCG Unresponsive, MCNA has the potential to render $26.5 \%$ of patients with CIS and $61.2 \%$ of patients with papillary tumors diseasefree for at least 1 year with an intact bladder. The higher efficacy noted in the true BCG-unresponsive cohort than was previously reported with all-comers, emphasizes the importance of having clearly defined criteria for clinical trials investigating new intravesical therapies after BCG failure.

\section{ACKNOWLEDGMENTS}

None.

\section{CONFLICT OF INTEREST}

AMK: Photocure; Telesta Therapeutics; Sanofi; Merck; Abbott Molecular; Theralase; Heat Biologics; Spectrum Pharmaceuticals; Oncogenex; Cepheid; FKD Industries; Pacific Edge.

\section{REFERENCES}

[1] Morales A, Herr H, Steinberg G, Given R, Cohen Z, Amrhein J, et al. Efficacy and safety of MCNA in patients with nonmuscle invasive bladder cancer at high risk for recurrence and progression after failed treatment with bacillus Calmette-Guerin. J Urol 2015;193(4):1135-43.

[2] Kamat AM, Sylvester RJ, Bohle A, Palou J, Lamm DL, Brausi $\mathrm{M}$, et al. Definitions, end points, and clinical trial designs for non-muscle-invasive bladder cancer: Recommendations from the international bladder cancer group. J Clin Oncol 2016;34(16):1935-44.

[3] Morales A, Eidinger D, Bruce AW. Intracavitary Bacillus Calmette-Guerin in the treatment of superficial bladder tumors. J Urol 1976;116(2):180-3. 
[4] Chin JL, Kadhim SA, Batislam E, Karlik SJ, Garcia BM, Nickel JC, et al. Mycobacterium cell wall: An alternative to intravesical bacillus Calmette Guerin (BCG) therapy in orthotopic murine bladder cancer. J Urol 1996;156(3): 1189-93.

[5] Filion MC, Phillips NC. Therapeutic potential of mycobacterial cell wall-DNA complexes. Expert Opin Investig Drugs 2001;10(12):2157-65.

[6] Morales A, Phadke K, Steinhoff G. Intravesical mycobacterial cell wall-DNA complex in the treatment of carcinoma in situ of the bladder after standard intravesical therapy has failed. J Urol 2009;181(3):1040-5.

[7] FDA Cellular T, and Gene Therapies Advisory Committee and Oncologic Drugs Advisory Committee. BLA 125593 Mycobacterium phlei Cell Wall-Nucleic Acid Complex 2015:1-49.

[8] Lerner SP, Dinney C, Kamat A, Bivalacqua TJ, Nielsen M, O'Donnell M, et al. Clarification of bladder cancer disease states following treatment of patients with intravesical BCG. Bladder Cancer (Amsterdam, Netherlands) 2015;1(1): 29-30.

[9] Chang SS, Boorjian SA, Chou R, Clark PE, Daneshmand $\mathrm{S}$, Konety BR, et al. Diagnosis and treatment of nonmuscle invasive bladder cancer: AUA/SUO guideline. J Urol 2016;196(4):1021-9.

[10] Babjuk M, Bohle A, Burger M, Capoun O, Cohen D, Comperat EM, et al. EAU guidelines on non-muscle-invasive urothelial carcinoma of the bladder: Update 2016. Eur Urol 2016. Epub ahead of print.

[11] Jarow JP, Lerner SP, Kluetz PG, Liu K, Sridhara R, Bajorin $\mathrm{D}$, et al. Clinical trial design for the development of new therapies for nonmuscle-invasive bladder cancer: Report of a food and drug administration and american urological association public workshop. Urology 2014;83(2):262-5.

[12] Steinberg G, Bahnson R, Brosman S, Middleton R, Wajsman Z, Wehle M. Efficacy and safety of valrubicin for the treatment of Bacillus Calmette-Guerin refractory carcinoma in situ of the bladder. The Valrubicin Study Group. J Urol 2000;163(3):761-7.

[13] Skinner EC, Goldman B, Sakr WA, Petrylak DP, Lenz HJ, Lee CT, et al. SWOG S0353: Phase II trial of intravesical gemcitabine in patients with nonmuscle invasive bladder cancer and recurrence after 2 prior courses of intravesical bacillus Calmette-Guerin. J Urol 2013;190(4):1200-4.

[14] McKiernan JM, Holder DD, Ghandour RA, Barlow LJ, Ahn JJ, Kates M, et al. Phase II trial of intravesical nanoparticle albumin bound paclitaxel for the treatment of nonmuscle invasive urothelial carcinoma of the bladder after bacillus Calmette-Guerin treatment failure. J Urol 2014;192(6):1633-8.

[15] Joudi FN, Smith BJ, O’Donnell MA, National BCGIPIG. Final results from a national multicenter phase II trial of combination bacillus Calmette-Guerin plus interferon alpha-2B for reducing recurrence of superficial bladder cancer. Urol Oncol 2006;24(4):344-8.

[16] Nativ O, Witjes JA, Hendricksen K, Cohen M, Kedar D, Sidi A, et al. Combined thermo-chemotherapy for recurrent bladder cancer after bacillus Calmette-Guerin. J Urol 2009;182(4):1313-7.

[17] Mmeje CO, Guo CC, Shah JB, Navai N, Grossman HB, Dinney $\mathrm{CP}$, et al. Papillary recurrence of bladder cancer at first evaluation after induction bacillus calmette-guerin therapy: Implication for clinical trial design. Eur Urol 2016;70(5):778-85.

[18] Oddens J, Brausi M, Sylvester R, Bono A, van de Beek C, van Andel G, et al. Final results of an EORTC-GU cancers group randomized study of maintenance bacillus Calmette-Guerin in intermediate- and high-risk Ta, T1 papillary carcinoma of the urinary bladder: One-third dose versus full dose and 1 year versus 3 years of maintenance. Eur Urol 2013;63(3):462-72. 